\title{
Fas expression in memory CD8+ T cell subsets augments cellular differentiation and effector function
}

\author{
Tori Yamamoto ${ }^{1 *}$, Anthony Leonardi ${ }^{1}$, Hui Liu², Ena Wang ${ }^{2}$, Luca Gattinoni ${ }^{3}$, Anthony Cruz ${ }^{4}$, Claudia Ouyang ${ }^{4}$, \\ Richard Siegel ${ }^{4}$, Nicholas Restifo ${ }^{1}$, Christopher A Klebanoff ${ }^{1}$ \\ From 30th Annual Meeting and Associated Programs of the Society for Immunotherapy of Cancer (SITC 2015) \\ National Harbor, MD, USA. 4-8 November 2015
}

Memory $\mathrm{CD}^{+} \mathrm{T}$ cells $\left(\mathrm{T}_{\mathrm{Mem}}\right.$ ) have the capacity to provide lifelong host protection against intracellular pathogens and cancer. Despite phenotypic and functional heterogeneity among $\mathrm{T}_{\mathrm{Mem}}$, the expression of Fas - a tumor necrosis family receptor (TNFR) superfamily member conventionally known as a death receptor - is held in common among all $\mathrm{T}_{\text {Mem }}$ subsets across multiple species. As Fas has been shown to mediate non-death signaling in other cell types, we set out to elucidate the role of Fas signaling in defined $\mathrm{T}_{\mathrm{Mem}}$ subsets, including $\mathrm{T}$ stem cell memory $\left(\mathrm{T}_{\mathrm{SCM}}\right)$, $\mathrm{T}$ central memory $\left(\mathrm{T}_{\mathrm{CM}}\right)$, and $\mathrm{T}$ effector memory $\left(\mathrm{T}_{\mathrm{EM}}\right)$. We found that augmenting Fas signaling in stimulated $\mathrm{T}_{\mathrm{SCM}}$ using an oligomerized form of its ligand FasL resulted in augmented cellular differentiation and a loss in IL-2 secretion capacity. Conversely, antibody blockade (anti-FasL) of Fas signaling in $\mathrm{T}_{\mathrm{CM}}$ retarded cellular differentiation both phenotypically and functionally. To genetically disentangle the pro-apoptotic and differentiation signals from Fas, we made use of a mutant Fas lacking a transmembrane cysteine residue (FasC194V) that is unable to undergo S-palmitoylation and aggregate efficiently in lipid rafts. Using transgenic mice expressing this C194V Fas construct on a Fas-deficient $l p r$ background, we found that FasC194V T $\mathrm{T}_{\mathrm{Mem}}$ can still undergo cellular differentiation in the absence of death signaling. In vivo, $\mathrm{T}_{\mathrm{Mem}}$ expanded with anti-FasL showed greater expansion, ontarget immunity and withheld differentiation. Additionally, in a relevant syngeneic model of current human $\mathrm{T}$ cell immunotherapy, $\mathrm{T}_{\mathrm{Mem}}$ cells expanded with anti-FasL and genetically engineered with an anti-CD19 chimeric antigen receptor (CAR) exhibited enhanced CAR expression,

${ }^{1}$ Center for Cancer Research, NCI/NIH, Bethesda, MD, USA

Full list of author information is available at the end of the article reduced differentiation, and augmented anti-lymphoma activity compared to controls. These studies demonstrate that Fas signaling promotes not only cell death but also $\mathrm{T}_{\mathrm{Mem}}$ effector differentiation, a finding that has implications for the design and execution of $\mathrm{T}$ cell-based immunotherapies in patients with cancer or infectious disease.

\section{Authors' details}

${ }^{1}$ Center for Cancer Research, NCI/NIH, Bethesda, MD, USA. Infectious Disease and Immunogenetics Section, Department of Transfusion Medicine, Clinical Center, NIH, Bethesda, MD, USA. ${ }^{3}$ Experimental Transplantation and Immunology Branch, $\mathrm{NCI} / \mathrm{NIH}$, Bethesda, MD, USA. ${ }^{4}$ National Institute of Arthritis and Musculoskeletal and Skin Diseases, NIH, Bethesda, MD, USA.

Published: 4 November 2015

\section{doi:10.1186/2051-1426-3-S2-P329}

Cite this article as: Yamamoto et al:: Fas expression in memory CD8+

$\mathrm{T}$ cell subsets augments cellular differentiation and effector function. Journal for ImmunoTherapy of Cancer 2015 3(Suppl 2):P329.

Submit your next manuscript to BioMed Central and take full advantage of:

- Convenient online submission

- Thorough peer review

- No space constraints or color figure charges

- Immediate publication on acceptance

- Inclusion in PubMed, CAS, Scopus and Google Scholar

- Research which is freely available for redistribution 